\title{
Direct Structural Identification and Quantification of the Split-Vacancy Configuration for Implanted Sn in Diamond
}

\author{
U. Wahl,${ }^{1,2, *}$ J. G. Correia, ${ }^{2}$ R. Villarreal, ${ }^{1}$ E. Bourgeois, ${ }^{3,4}$ M. Gulka, ${ }^{3}$ M. Nesládek, ${ }^{3,4}$ A. Vantomme,,${ }^{1}$ and L. M. C. Pereira ${ }^{1}$ \\ ${ }^{1}$ KU Leuven, Quantum Solid-State Physics, 3001 Leuven, Belgium \\ ${ }^{2}$ Centro de Ciências e Tecnologias Nucleares, Departamento de Engenharia e Ciências Nucleares, Instituto Superior Técnico, Universidade \\ de Lisboa, 2695-066 Bobadela LRS, Portugal \\ ${ }^{3}$ Institute for Materials Research (IMO), Hasselt University, 3590 Diepenbeek, Belgium \\ ${ }^{4}$ IMOMEC division, IMEC, 3590 Diepenbeek, Belgium \\ (Received 6 November 2019; revised 28 May 2020, accepted 1 July 2020)
}

\begin{abstract}
We demonstrate formation of the ideal split-vacancy configuration of the Sn-vacancy center upon implantation into natural diamond. Using $\beta^{-}$emission channeling following low fluence ${ }^{121} \mathrm{Sn}$ implantation $\left(2 \times 10^{12}\right.$ atoms $/ \mathrm{cm}^{2}, 60 \mathrm{keV}$ ) at the ISOLDE facility at CERN, we directly identified and quantified the atomic configurations of the Sn-related centers. Our data show that the split-vacancy configuration is formed immediately upon implantation with a surprisingly high efficiency of $\approx 40 \%$. Upon thermal annealing at $920^{\circ} \mathrm{C} \approx 30 \%$ of $\mathrm{Sn}$ is found in the ideal bond-center position. Photoluminescence revealed the characteristic $\operatorname{Sn} V$ line at $621 \mathrm{~nm}$, with an extraordinarily narrow ensemble linewidth $(2.3 \mathrm{~nm})$ of near-perfect Lorentzian shape. These findings further establish the $\mathrm{Sn} V$ center as a promising candidate for single photon emission applications, since, in addition to exceptional optical properties, it also shows a remarkably simple structural formation mechanism. DOI: 10.1103/PhysRevLett.125.045301
\end{abstract}

Point defects in diamond are being intensively investigated for their applications in quantum information science, as well as for quantum metrology [1]. So far, the negatively charged nitrogen-vacancy center $(\mathrm{N} V)$ has been the most studied solid-state qubit [1-6] thanks to its efficient optical spin polarization, spin-state dependent fluorescence, and long spin coherence time at room temperature (RT). Owing to their superior optical properties such as their higher Debye-Waller factor, the negatively charged group-IV-vacancy centers, mainly $\mathrm{Si} V$ [7-9] and $\mathrm{Ge} V$ [10-11] are intensively studied, however, these centers have short spin coherence times. Lately $\mathrm{Sn} V$ [12-20] and $\mathrm{Pb} V$ [21-22] have emerged as highly interesting types of point defects for diamondbased quantum nanophotonics and quantum communication, due to expected longer coherence times and this at higher temperatures than $\mathrm{Si} V$, as was recently reviewed in Refs. [23-24]. Nonetheless many key properties and especially the formation mechanism and local configuration of these defects are largely unexplored.

It is generally accepted that, whereas the $\mathrm{N}$ atom in the $\mathrm{N} V$ center occupies a substitutional carbon site, the groupIV-vacancy centers form a so-called split-vacancy configuration, where the group IV impurity atom sits in between two adjacent $\mathrm{C}$ vacancies in a position that would correspond to a bond-centered (BC) site in an unperturbed lattice (Fig. 1). This configuration was first proposed for the $\mathrm{Si}$ impurity by Goss et al. [25-27] based on ab-initio local density calculations, and later also extended by theory to the other group IV elements $\mathrm{Ge}, \mathrm{Sn}$, and $\mathrm{Pb}$ [22,26,28-29].

Experimentally, however, the group-IV-vacancy structural configurations have only been indirectly supported, e.g., from the number of optical emission lines and their polarization and magnetic field splitting [19-20,30-31]. Techniques for direct, detailed, and quantitative characterization of the structure of these defects are especially important for the quantum technology field, since the superior optical properties of the group-IV-vacancy centers are to a large extent a consequence of their $D_{3 \mathrm{~d}}$ inversion symmetry rather than $C_{3 \mathrm{v}}$, as in the case of $\mathrm{N} V$. For a split-vacancy configuration, however, $D_{3 \mathrm{~d}}$ symmetry will only be realized if the impurity is exactly centered within the divacancy, which corresponds to the ideal BC position. Currently the most used technique for creating group-IV-vacancy centers is ion implantation [7-14,17-24,31-32]. The fractions of implanted impurities that form the relevant optically active color centers can then be obtained from comparing the number of centers per area found with confocal luminescence microscopy to the implanted fluence. For undoped diamond, this yielded formation efficiencies of optically active $\mathrm{Sn} V$ centers around $5 \%$ [12], 1\%-2\% [13], 0.7\% [17], and 2.5\% [18] of implanted atoms only. By carefully engineering Sn implantations into phosphorus-doped diamond, the highest optical activation for $\mathrm{Sn} V$ reported so far [18] is $8.6 \%$ after annealing at $1200^{\circ} \mathrm{C}$. Because of the lack of structural characterization methods it is unknown whether higher activation yields are inhibited because the relevant $\operatorname{Sn} V$ centers are not formed or because they are formed but in an optically inactive state or their optical activity is quenched.

In this letter, we provide direct lattice location measurements for implanted radioactive ${ }^{121} \mathrm{Sn}$ in diamond using the $\beta^{-}$emission channeling (EC) technique. EC is uniquely suited to characterize and quantify the possible point defect configurations via the lattice sites of the impurity atoms. In particular, this structural technique is able to work at the low implantation fluences $\left(\approx 10^{12} \mathrm{~cm}^{-2}\right)$ that are often used to produce quantum color centers in diamond. We show that a surprisingly large fraction of $\mathrm{Sn}(\approx 40 \%)$ already forms $\mathrm{Sn} V$ centers directly upon implantation without any annealing. Additionally, we confirm formation of $\operatorname{Sn} V$ optically by confocal photoluminescence (PL) resulting from long-lived 

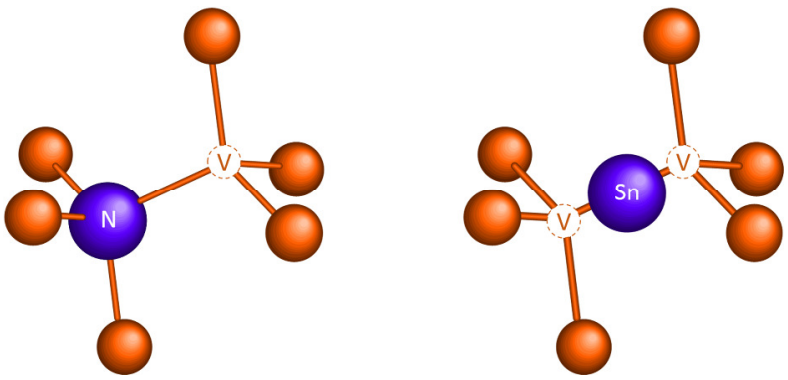

FIG. 1. The two major structures that are generally considered for impurity-vacancy complexes in diamond. It is assumed that with a vacancy $V$ next to it the $\mathrm{N}$ impurity remains in a substitutional site (left), while for Sn the so-called split-vacancy configuration (right) has been proposed $[25,28]$ : the Sn atom moves from its substitutional site to a position midway between the two adjacent vacancies, which geometrically coincides with the so-called bond-center position in an undisturbed lattice.

${ }^{121 \mathrm{~m}} \mathrm{Sn}$ in the same sample. Following annealing at $920^{\circ} \mathrm{C}$, both EC and PL find $\mathrm{Sn} V$ centers in a single, well-defined atomic configuration, leading to a remarkably narrow luminescence linewidth.

The basic concept of the $\beta^{-} \mathrm{EC}$ technique is that the lattice sites of implanted radioactive isotopes in single crystals are probed via the channeling of emitted beta decay particles [33-35]. The underlying physical effect is the guidance of $\beta^{-}$ particles by the crystal potential, which strongly depends on the lattice site from which the particle originated. This leads to an angular dependence of the $\beta^{-}$emission yield in the vicinity of major crystallographic directions, which is recorded by a two-dimensional position-sensitive detector [36], thus providing patterns that are characteristic for the probe atom lattice location in the sample. The fact that EC is based on the detection of decay particles from radioactive probes makes it a factor of $\approx 10^{4}$ more efficient [35] than conventional lattice location techniques using external ion beams. The capability of EC to identify impurities on the BC site in a diamond lattice structure following implantations at the $10^{12}-10^{13} \mathrm{~cm}^{-2}$ level has been previously demonstrated, e.g., in $\mathrm{Ge}$ for ${ }^{121} \mathrm{Sn}$ [37] and for the transition metals ${ }^{59} \mathrm{Fe},{ }^{67} \mathrm{Cu}$ and ${ }^{111} \mathrm{Ag}$ [38].

For determining the lattice location of Sn, we have used the short-lived radioactive isotope ${ }^{121} \mathrm{Sn}$, which decays with a half-life of $t_{1 / 2}=27.06 \mathrm{~h}$ via $\beta^{-}$emission $\left(\beta^{-}\right.$endpoint energy $400 \mathrm{keV}$, average $116 \mathrm{keV}$ ) into stable ${ }^{121} \mathrm{Sb}$, while the detected PL signal from $\mathrm{Sn} V$ resulted from the metastable, long-lived isomeric state ${ }^{121 \mathrm{~m}} \mathrm{Sn}\left(t_{1 / 2}=55 \mathrm{y}\right)$. Both ${ }^{121} \mathrm{Sn}$ in its ground and isomeric state were produced and, due to their near identical mass, simultaneously implanted at CERN's ISOLDE on-line isotope separator facility [39-41]. The sample was a natural type IIa diamond (Dutch Diamond Technologies, $3 \times 3 \mathrm{~mm}^{2}$ size, thickness $300 \mu \mathrm{m}$ ), with the surface cut and polished $8^{\circ}$ from a $<110>$ direction. The implanted fluence of $60 \mathrm{keV}{ }^{121} \mathrm{Sn}+{ }^{121 \mathrm{~m}} \mathrm{Sn}$ was $2.3 \times 10^{12}$ at$\mathrm{oms} / \mathrm{cm}^{2}$, into a beam spot of $1 \mathrm{~mm}$ diam (average current 3 pA). According to SRIM simulations [42], this results in a projected range and straggling of $211 \pm 40 \AA$, Sn peak concentration $2.3 \times 10^{18}$ atoms $/ \mathrm{cm}^{3}$, mean $\mathrm{Sn}-\mathrm{Sn}$ distances larger than $76 \AA$, and each implanted atom initially creating $\approx 430$ lattice vacancies. By obtaining the number of implanted ${ }^{121} \mathrm{Sn}$ atoms from the measured $\beta^{-}$count rate, the ratio of ${ }^{121} \mathrm{Sn}:{ }^{121 \mathrm{~m}} \mathrm{Sn}$ was estimated to be $\approx 44: 56$ [43].

In order to derive the fractions of ${ }^{121} \mathrm{Sn}$ on different lattice sites, the angular-dependent experimental $\beta^{-}$emission yields were fitted by theoretical patterns corresponding to ${ }^{121} \mathrm{Sn}$ emitter atoms on up to two different lattice sites. The theoretical $\beta^{-}$patterns were calculated by means of the socalled "many-beam" formalism outlined in Refs. [33-34,4347]; general aspects of the fit procedure are described in Refs. [34-36,48]. The major lattice sites in the diamond structure that were considered in the simulations are shown in the supplemental material [43]. They included substitutional $(S)$ and $\approx 250$ interstitial sites which are obtained by displacing from the $S$ position along $<111\rangle,<100\rangle$, or $<110>$ directions in steps of $\approx 0.04 \AA$. Besides such static displacements, we also calculated the expected yields for sites that are spread around $S$ and BC positions with Gaussian probability distributions of larger root mean square (rms) than expected from thermal vibrations alone, which for a diamond Debye temperature of $1860 \mathrm{~K}$ [49] is $u_{1}\left({ }^{121} \mathrm{Sn}\right)=0.0337 \AA$ [43].

Figures 2(a)-2(d) show the angular distribution of $\beta^{-}$particles emitted around the $\langle 110\rangle,\langle 211\rangle,<100\rangle$ and $<111\rangle$ directions in the as-implanted state, while Figs 3(a)-3(d) are following $920^{\circ} \mathrm{C}$ annealing for $10 \mathrm{~min}$ in vacuum. The fact that all axial and planar directions exhibit channeling effects shows that a large fraction of emitter atoms occupies sites close to the substitutional positions. Note that the anisotropy of the channeling effects roughly doubles upon annealing, which is a clear indication of damage annealing and/or a change of the configuration of $\mathrm{Sn}$ in the lattice.

The experimental patterns were first fitted using one-site fits, i.e., allowing for only one of the calculated positions in the lattice to be occupied. A detailed description of the analysis procedure can be found in the supplemental material [43]. However, neither in the as-implanted nor in the annealed state simple ideal substitutional sites provided the best one-site fits; these were rather obtained for sites that are displaced from the ideal $S$ sites: in the as-implanted state displacements of $30 \%-50 \%(0.23-0.39 \AA)$ of the distance from $S$ to BC sites yielded chi square $\left(\chi^{2}\right)$ improvements up to $44 \%$ (average for all four patterns $24 \%$ ) compared to ideal $S$ sites; following $920^{\circ} \mathrm{C}$ annealing displacements of $10 \%$ $30 \%\left(0.08-0.23 \AA\right.$ ) improved $\chi^{2}$ by up to $19 \%$ (average $9 \%$ ). However, $\chi^{2}$ further improved by allowing $\mathrm{Sn}$ on two different lattice sites in the fit procedure. In the as-implanted state the overall best fit was obtained for a combination of sites which are spread around the $S$ and BC positions with rms displacements of $u_{1}\left({ }^{121} \mathrm{Sn}\right)$ of 0.18 and $0.11 \AA$, respectively. While this combination of sites led to improvements up to $45 \%$ (average $26 \%$ ) compared to ideal $S$ sites, this result was only slightly better than when one statically displaced site was considered; it can therefore not be discerned whether in the as-implanted state a single site located in between $S$ and BC or a combination of $S$ and BC sites with larger rms amplitudes prevails. In contrast, in the 


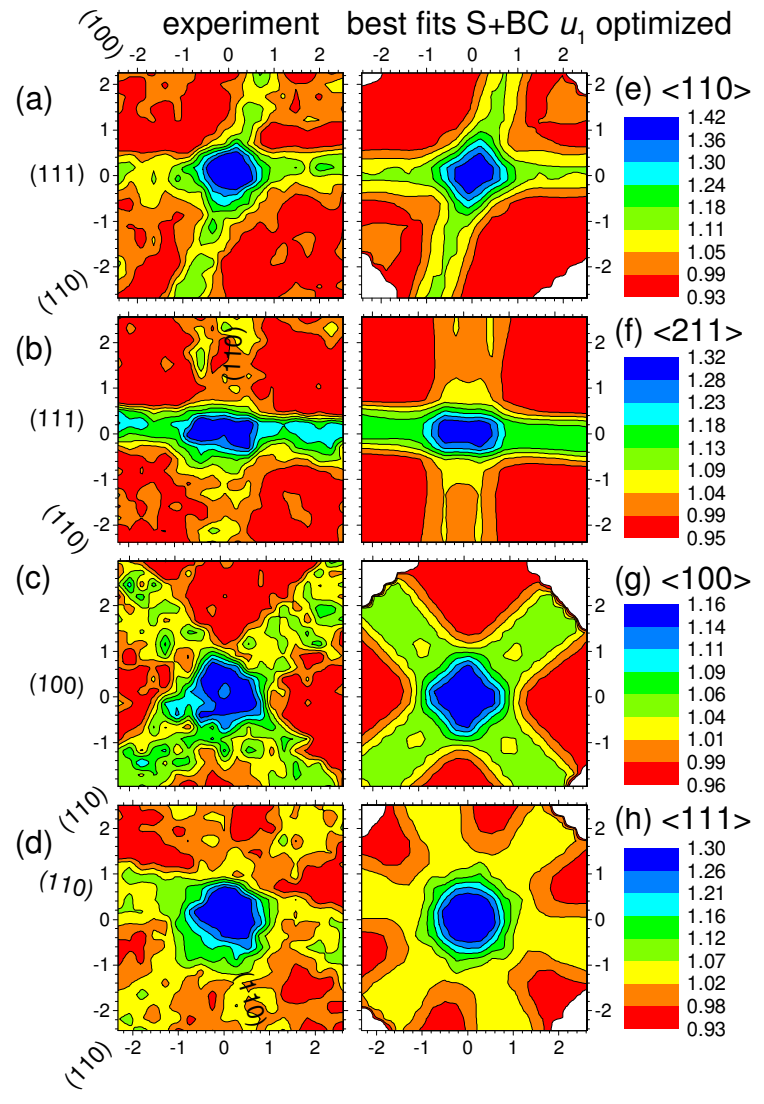

FIG. 2. (a)-(d): Experimental $\beta^{-}$emission channeling patterns from ${ }^{121} \mathrm{Sn}$ in diamond around the $\langle 110\rangle,\langle 211\rangle,\langle 100\rangle$, and $\langle 111\rangle$ directions in the as-implanted state. The plots (e)-(h) are the corresponding best fits of theoretical patterns considering $63 \%$ on substitutional and $41 \%$ on bond-center sites with rms $u_{1}\left({ }^{121} \mathrm{Sn}\right)$ of 0.18 $\AA$ and $0.11 \AA$, respectively. Note that due to the unusual surface cut of the diamond, it could not be oriented in all cases in such a way that the calculated patterns $\left[ \pm 3^{\circ}\right.$ with $(110)$ plane horizontal $]$ cover the whole range of measured angles. The area in the best-fit patterns (e)-(h) which is not covered is shown in white.

$920^{\circ} \mathrm{C}$ annealed state the best fit was clearly obtained for a combination of ideal $S$ and BC sites, resulting in significant $\chi^{2}$ improvements (up to $24 \%$, average $12 \%$ ) compared to $S$ sites only. The theoretical patterns that correspond to the best fits are shown in Figs 2(e)-2(h) and 3(e)-3(h) and match the experimental results in considerable detail.

For the analysis of the as-implanted state we adopted the interpretation of two occupied sites; in this case $(63 \pm 6) \%$ of ${ }^{121} \mathrm{Sn}$ is assigned to $S$ and $(41 \pm 5) \%$ to BC sites, however, as was already mentioned, both sites are not the ideal ones but subject to Gaussian spreads. Using a single site located in between $S$ and BC would result in a lower fraction of $81 \%$ only. The situation following $920^{\circ} \mathrm{C}$ annealing is characterized by two major changes: (a) BC as well as $S$ sites become ideal, (b) fractions are now $f_{S}=(79 \pm 6) \%$ and $f_{\mathrm{BC}}=(32 \pm 5) \%$, i.e. some of the ${ }^{121} \mathrm{Sn}$ on $\mathrm{BC}$ has been converted to $S$ sites. Both effects together are responsible for the pronounced change in the anisotropy of the channeling patterns upon annealing. The sum $f_{\text {sum }}=f_{S}+f_{\mathrm{BC}}$ of the two fitted fractions reaches values slightly above $100 \%$, which is related to an

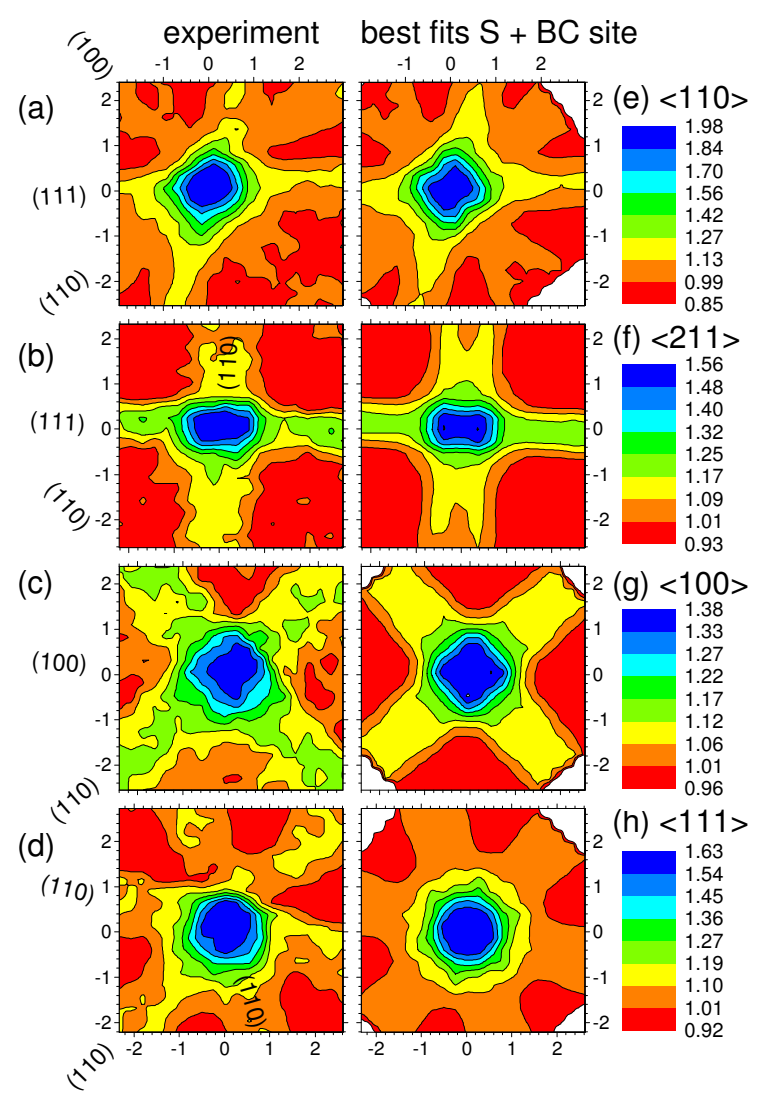

FIG. 3. (a)-(d): Experimental $\beta^{-}$emission channeling patterns from ${ }^{121} \mathrm{Sn}$ following $920^{\circ} \mathrm{C}$ annealing. The plots (e)-(h) are the corresponding best fits of theoretical patterns considering $79 \%$ on ideal substitutional and $32 \%$ on ideal bond-center sites.

overestimation of the background correction from scattered electrons, as is explained in the supplemental material [43].

Confocal PL spectra (Fig. 4) following annealing at $920^{\circ} \mathrm{C}$ show the prominent first order Raman peak at 573 $\mathrm{nm}$ and also the weaker second order Raman line at $612 \mathrm{~nm}$. Focusing below the surface, the characteristic features of the $\mathrm{N} V^{0}$ zero phonon line (ZPL) at $575 \mathrm{~nm}$ and the $\mathrm{N} V$ ZPL at $636 \mathrm{~nm}$ and associated phonon side bands at longer wavelengths are visible, reflecting the presence of nitrogenassociated defects in the natural IIa diamond. Detailed confocal scanning of the sample [43] showed that in the spots where low $\mathrm{N} V$ signals are found, close to the implanted surface a strong, sharp peak is detected at $621.3 \mathrm{~nm}$, corresponding to the well-known ZPL of $\mathrm{Sn} V$ [12-21,23-24,29]. Fitting of this line using a Lorentzian [43] shows a full width half maximum (FWHM) of $2.3 \mathrm{~nm}$, the narrowest RT linewidth reported to date for the ZPL of an ensemble of $\operatorname{Sn} V$ [12-16,19], cf. Ref. [43] for an overview on linewidths. The near ideal Lorentzian shape [43] implies a very narrow distribution of single center emission lines in the whole ensemble. The 621-nm line is accompanied by much weaker peaks at 631 and $643 \mathrm{~nm}$, in good agreement with Refs. [12-14,18]. The peaks at 621, 631, and $643 \mathrm{~nm}$ weaken as the focus is set deeper into the sample, away from the implanted surface. A sharp PL line at $581.4 \mathrm{~nm}$ (FWHM 1.5 


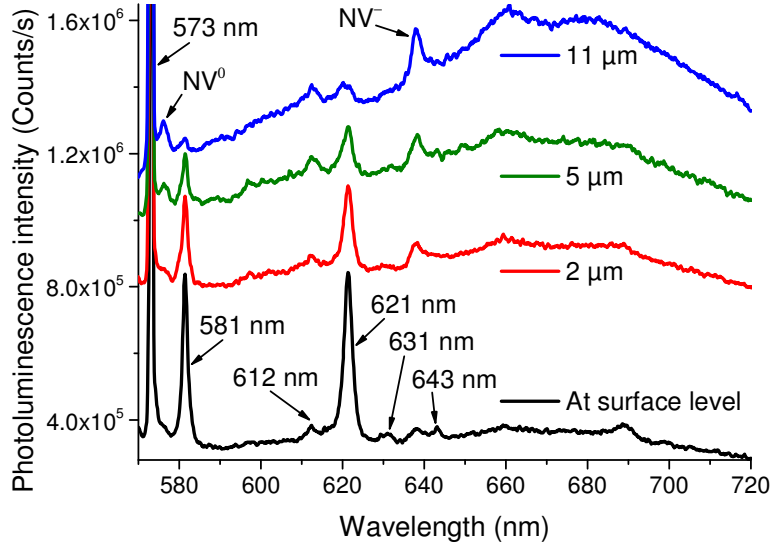

FIG. 4. Confocal photoluminescence spectra from the diamond sample implanted with ${ }^{121} \mathrm{Sn}+{ }^{121 \mathrm{~m}} \mathrm{Sn}$ ions after annealing at $920^{\circ} \mathrm{C}$ and following the decay of ${ }^{121} \mathrm{Sn}$ to ${ }^{121} \mathrm{Sb}$, i.e. $\mathrm{SnV}$ related lines result from long-lived ${ }^{121 \mathrm{~m}} \mathrm{Sn}$. The spectra were recorded at room temperature under $532 \mathrm{~nm}$ laser excitation with the focus at various depths. Note that the depth resolution is $\approx 4 \mu \mathrm{m}$ due to the Rayleigh range of the used objective with numerical aperture 0.95 .

$\mathrm{nm})$ is additionally observed close to the surface. This line could correspond to the L1 center, a bright defect of unknown nature presenting a narrow ZPL at $581.7 \mathrm{~nm}$ (reported FWHM $<1.4 \mathrm{~nm}$ ), which has been detected in diamond implanted with a mixture of molecular ions of mass 27 and annealed at $800^{\circ} \mathrm{C}$ [32,50]. For a discussion of the possible sources for this line we refer to the supplemental material [43].

From the combined results of EC and PL, we obtain unique insight in the structure and formation mechanism of $\mathrm{Sn} V$ centers. First of all we have provided direct evidence that considerable amounts of $\mathrm{Sn}$ atoms implanted in diamond are found on lattice sites that correspond to the split-vacancy configuration of the $\operatorname{Sn} V$ center. The microscopic model so far assumed for $\operatorname{Sn} V$ centers and their $D_{3 \mathrm{~d}}$ symmetry is thus fully supported by our results, obtained with a technique that directly probes structure.

Second, our EC results show that the fraction of implanted Sn on BC sites is surprisingly high, about an order of magnitude above the formation yield of optically active $\operatorname{Sn} V$ centers reported from confocal PL [12-13,1718]. If these previous studies should have achieved the same structural formation yield of $\mathrm{BC} \mathrm{Sn}$, this would mean that $\approx 90 \%$ of the $\mathrm{Sn} V$ centers were optically inactive at $621 \mathrm{~nm}$, e.g. because they exist in a different charge state than $\operatorname{Sn} V$, such as $\operatorname{Sn} V^{0}$, do not possess the right $D_{3 \mathrm{~d}}$ symmetry, or are prevented from being optically active by some yet unknown mechanism, e.g., H passivation [51]. In this respect we note that all previous Sn implantation studies (cf. list in Ref. [43]) have used synthetic diamond grown by chemical vapor deposition (CVD), which tends to be rich in $\mathrm{H}$, while we used a natural diamond. By pre-doping CVD diamond with the deep donor phosphorus prior to $\mathrm{Sn}$ implantation, Lühmann et al. recently achieved optical activation of $\operatorname{Sn} V$ in the $8 \%-9 \%$ range [18]. This was attributed to vacancies in diamond becoming negatively charged $V_{\mathrm{C}}{ }^{-}$in the presence of donors, thus being prevented from agglomerating by Coulomb repulsion and leading to "a more efficient formation of $\mathrm{Sn} V$ together with a reduced formation of competing centres" [18]. Since EC can quantify the presence of ${ }^{121} \mathrm{Sn}$ on $\mathrm{BC}$ sites from the measurement of a single $<110>$, $<211>$ or $<100>$ emission pattern, which requires an implanted fluence around $1 \times 10^{11} \mathrm{~cm}^{-2}$ only, it seems possible to test in future experiments whether $\mathrm{P}$ doping directly changes the structural formation efficiency of $\operatorname{Sn} V$, as suggested in Ref. [18], or rather favors its activation as an optical center.

Third, our studies shed light on the creation of $\operatorname{Sn} V$ centers during ion implantation and the role of thermal annealing. The centers are clearly already formed in large quantities during the implantation process and the subsequent cool down of the thermal spike caused by the collision cascade, although in a perturbed configuration that is attributed to nearby radiation damage such as vacancies and interstitials. Considering the formation and binding energies for Sn defects in diamond calculated by Goss et al. [26], from a thermodynamic point of view this can be understood as follows, using similar arguments as presented for $\mathrm{Sn}$ in $\mathrm{Ge}$ [38]. Isolated substitutional $\mathrm{Sn}$ is predicted to have the lowest heat of formation of all Sn defects and should hence be the dominant species; however, when it encounters an additional vacancy it becomes energetically favorable to form $\operatorname{Sn} V$ and change its lattice site to the $\mathrm{BC}$ position. The energy gain associated with this process is predicted in the 8-9 eV range [26]. While the required vacancies are abundantly available and also mobile during the cool-down of the thermal spike following implantation of each ion, $\operatorname{Sn} V$ formation has to compete with the annihilation of vacancies by carbon interstitials. As our results show, thermal annealing is not required in order to bring additional vacancies towards the implanted $\mathrm{Sn}$ (as is sometimes wrongly assumed in analogy to $\mathrm{N} V$ ). The annealing process serves two purposes: (a) restoring the optical properties of diamond after the implantation, (b) restructuring the $\operatorname{Sn} V$ center into its ideal configuration where $\mathrm{Sn}$ resides on unperturbed $\mathrm{BC}$ sites. To these effects, diffusion of vacancies is still essential for removing defects, in particular also from the immediate $\operatorname{Sn} V$ neighborhood, which has recently been studied using a theoretical approach [52]. It was predicted that $V_{\mathrm{C}^{-}} \mathrm{Sn} V$ complexes are thermodynamically more stable than their components, i.e., isolated $\operatorname{Sn} V$ and vacancies $V_{\mathrm{C}}$, which are both present in implanted samples. The formation of such complexes may therefore be responsible for the larger rms displacements of $\mathrm{Sn}$ on BC sites that we observed in the as-implanted state of our sample. The activation energy for breaking up $V_{\mathrm{C}}-\mathrm{Sn} V$ complexes was calculated as $E_{\mathrm{A}}=2.4-3.4 \mathrm{eV}$ (depending on the charge state), with an attempt frequencies of $v_{0} \approx 10^{14} \mathrm{~s}^{-1}$. Using simple Arrhenius reaction rates one estimates that the expected lifetime $\tau=1 /\left[v_{0} \exp \left(-E_{\mathrm{A}} / k_{\mathrm{B}} T\right)\right]$ of the complexes at $920^{\circ} \mathrm{C}$ is only $0.14 \mathrm{~ms}$ to $2.3 \mathrm{~s}$, before the $V_{\mathrm{C}}$ diffuses away, leaving behind undisturbed $\operatorname{Sn} V$. 
Fourth, the PL characterization performed on the $920^{\circ} \mathrm{C}$ annealed sample revealed ensembles of ${ }^{121 \mathrm{~m}} \mathrm{Sn} V$ ZPLs with near-perfect Lorentzian shape and with the narrowest RT ensemble linewidth reported so far $(2.3 \mathrm{~nm}$, just slightly larger than a recently found single center linewidth of 1.98 $\mathrm{nm}$ [20]). It is thus clearly possible to produce entire ensembles of ion implanted $\operatorname{Sn} V$ centers with a narrow spread of configurations without the need for annealing much higher than $900^{\circ} \mathrm{C}$. However, the exact conditions that are required to achieve ensembles of optically active $\operatorname{Sn} V$ centers with such a narrow linewidth are still unclear, although low implantation fluences seem to be crucial [43]. While in our natural diamond sample optically active $\operatorname{Sn} V$ centers were only detected in areas with low PL signals from $\mathrm{N} V$ centers, whenever an ensemble of $\mathrm{Sn} V$ lines was found, it exhibited a narrow linewidth [43].

Last but not least, the EC technique has been found well suited to investigate the structural nature of ensembles of implanted optical centers in diamond. Suitable EC probes for further studies of group-IV-vacancy centers could be ${ }^{31} \mathrm{Si}$ $\left(t_{1 / 2}=157 \mathrm{~min}\right),{ }^{75} \mathrm{Ge}(82.8 \mathrm{~min})$, and ${ }^{209} \mathrm{~Pb}(3.2 \mathrm{~h})$; for other elements of interest as single photon emitters also suitable $\beta^{-}$emitters exist, e.g., ${ }^{27} \mathrm{Mg},{ }^{45} \mathrm{Ca},{ }^{89} \mathrm{Sr},{ }^{65} \mathrm{Ni}$, or the noble gases ${ }^{6} \mathrm{He},{ }^{23} \mathrm{Ne},{ }^{41} \mathrm{Ar},{ }^{87} \mathrm{Kr}$, and ${ }^{135} \mathrm{Xe}$. In many cases EC could be ideally complemented by radiotracer PL, which allows the direct chemical identification of PL lines via correlating their change in intensity with the half-life of radioactive decay [40].

We thank the ISOLDE collaboration for providing radioactive Sn beams, and P. Siyushev for the measurement shown in Fig. S13(b) of the supplemental material. This work was funded by KU Leuven (SF/18/008), the Portuguese Foundation for Science and Technology (FCT, CERN/FIS-PAR/0005/2017), and the FWO Vlaanderen (G0E7417N, S004018N). The EU Horizon 2020 Framework supported ISOLDE beam times through grant agreement 654002 (ENSAR2), use of KU Leuven facilities through 824096 (RADIATE) and U. Hasselt through 820394 (ASTERIQS).

* Corresponding author.

uwahl@ctn.tecnico.ulisboa.pt

[1] F. Lenzini, N. Gruhler, N. Walter, and W. H. P. Pernice, Diamond as a platform for integrated quantum photonics, Adv. Quantum Technol. 1, 1800061 (2018).

[2] A. Gruber, A. Dräbenstedt, C. Tietz, L. Fleury, J. Wrachtrup, and C. von Borczyskowski, Scanning confocal optical microscopy and magnetic resonance on single defect centers, Science 276, 2012 (1997)

[3] M. W. Doherty, N. B. Manson, P. Delaney, F. Jelezko, J. Wrachtrup, and L. C. Hollenberg, The nitrogen-vacancy colour centre in diamond, Phys. Rep. 528, 1 (2013).

[4] B. Hensen, H. Bernien, A. E. Dreau, A. Reiserer, N. Kalb, M. S. Blok, J. Ruitenberg, R. F. L. Vermeulen ,R. N. Schouten, C. Abellan, W. Amaya, V. Pruneri, M. W. Mitchell, M. Markham, D. J. Twitchen, D. Elkouss, S. Wehner, T. H. Taminiau, and R. Hanson, Loophole-free Bell inequality violation using electron spins separated by 1.3 kilometres, Nature (London) 526, 682 (2015).

[5] S. Schmitt, T. Gefen, F. M. Stürner, T. Unden, G. Wolff, C. Müller, J. Scheuer, B. Naydenov, M. Markham, S. Pezzagna, J. Meijer, I. Schwarz, M. Plenio, A. Retzker, L. P. McGuinness, and F. Jelezko, Submillihertz magnetic spectroscopy performed with a nanoscale quantum sensor, Science 356, 832 (2017).

[6] C. E. Bradley, J. Randall, M. H. Abobeih, R. C. Berrevoets, M. J. Degen, M. A. Bakker, M. Markham, D. J. Twitchen, and T. H. Taminiau, A ten-qubit solid state spin register with quantum memory up to one minute, Phys. Rev. X 9, 031045 (2019).

[7] A. Sipahigil, R. E. Evans, D. D. Sukachev, M. J. Burek, J. Borregaard, M. K. Bhaskar, C. T. Nguyen, J. L. Pacheco, H. A. Atikian, C. Meuwly, and R. M. Camacho, An integrated diamond nanophotonics platform for quantum optical networks, Science 354, 847 (2016).

[8] D. D. Sukachev, A. Sipahigil, C. T. Nguyen, M. K. Bhaskar, R. E. Evans, F. Jelezko, and M. D. Lukin, Silicon-vacancy spin qubit in diamond: A quantum memory exceeding $10 \mathrm{~ms}$ with single-shot state readout, Phys. Rev. Lett. 119, 223602 (2017).

[9] T. Schröder, M. E. Trusheim, M. Walsh, L. Li, J. Zheng, M.. Schukraft, A. Sipahigil, R. E. Evans, D. D. Sukachev, C. T. Nguyen, J. L. Pacheco, R. M. Camacho, E. S. Bielejec, M. D. Lukin, and D. Englund, Scalable focused ion beam creation of nearly life time limited single quantum emitters in diamond nanostructures, Nat. Commun. 8, 15376 (2017).

[10] T. Iwasaki, F. Ishibashi, Y. Miyamoto, Y. Doi, S. Kobayashi, T. Miyazaki, K. Tahara, K. D. Jahnke, L. J. Rogers, B. Naydenov, F. Jelezko, S. Yamasaki, S. Nagamachi, T. Inubushi, N. Mizuochi, and M. Hatano, Germanium-vacancy single color centers in diamond, Sci. Rep. 5, 12882 (2015).

[11] M. K. Bhaskar, D. D. Sukachev, A. Sipahigil, R. E. Evans, M. J. Burek, C. T. Nguyen, L. J. Rogers, P. Siyushev, M. H. Metsch, H. Park, F. Jelezko, M. Loncar, and M. D. Lukin, Quantum nonlinear optics with a germanium-vacancy color center in a nanoscale diamond waveguide, Phys. Rev. Lett. 118, 223603 (2017).

[12] S. D. Tchernij, T. Herzig, J. Forneris, J. Küpper, S. Pezzagna, P. Traina, E. Moreva, I. P. Degiovanni, G. Brida, N. Skukan, M. Genovese, M. Jakšić, J. Meijer, and P. Olivero, Singlephoton-emitting optical centers in diamond fabricated upon Sn implantation, ACS Photonics 4, 2580 (2017).

[13] T. Iwasaki, Y. Miyamoto, T. Taniguchi, P. Siyushev, M. H. Metsch, F. Jelezko, and M. Hatano, Tin-vacancy quantum emitters in diamond, Phys. Rev. Lett. 119, 253601 (2017).

[14] M. Alkahtani, I. Cojocaru, X. Liu, T. Herzig, J. Meijer, J. Küpper, T Lühmann, A. V. Akimov, and P. R. Hemmer, Tinvacancy in diamonds for luminescent thermometry, Appl. Phys. Lett. 112, 241902 (2018).

[15] E. A. Ekimov, S. G. Lyapin, and M. V. Kondrin, Tin-vacancy color centers in micro- and polycrystalline diamonds synthesized at high pressures, Diamond Relat. Mater. 87, 223 (2018).

[16] Y. N. Palyanov, I. N. Kupriyanov, and Y. M. Borzdov, Highpressure synthesis and characterization of Sn-doped single crystal diamond, Carbon 143, 769 (2019).

[17] A. E. Rugar, C. Dory, S. Sun, and J. Vuckovic, Characterization of optical and spin properties of single tin- 
vacancy centers in diamond nanopillars, Phys. Rev. B 99, 205417 (2019).

[18] T. Lühmann, R. John, R. Wunderlich, J. Meijer, and S. Pezzagna, Coulomb-driven single defect engineering for scalable qubits and spin sensors in diamond, Nat. Commun. 10, 4956 (2019).

[19] M. E. Trusheim et al., Transform-limited photons from a coherent tin-vacancy spin in diamond, Phys. Rev. Lett. 124, 023602 (2020).

[20] J. Görlitz, D. Herrmann, G. Thiering, P. Fuchs, M. Gandil, T. Iwasaki, T. Taniguchi, M. Kieschnick, J. Meijer, M. Hatano, A. Gali, and C. Becher, Spectroscopic investigations of negatively charged tin-vacancy centres in diamond, New J. Phys. 22, 013048 (2020).

[21] S. D. Tchernij, T. Lühmann, T. Herzig, J. Küpper, A. Damin, S. Santonocito, M. Signorile, P. Traina, E. Moreva, F. Celegato, S. Pezzagna, I. P. Degiovanni, P. Olivero, M. Jakšić, J. Meijer, P. M. Genovese, and J. Forneris, Single-photon emitters in lead-implanted single-crystal diamond, ACS Photonics 5, 4864 (2018).

[22] M. E. Trusheim, N. H. Wan, K. C. Chen, C. J. Ciccarino, J. Flick, R. Sundararaman, G. Malladi, E. Bersin, M. Walsh, B. Lienhard, H. Bakhru, P. Narang, and D. Englund, Lead-related quantum emitters in diamond, Phys. Rev. B 99, 075430 (2019).

[23] D. Chen, N. Zheludev, and W. B. Gao, Building blocks for quantum network based on group-IV split-vacancy centers in diamond, Adv. Quantum Technol. 3, 1900069 (2019).

[24] C. Bradac, W. Gao, J. Forneris, M. E. Trusheim, and I. Aharonovich, Quantum nanophotonics with group IV defects in diamond, Nat. Commun. 10, 5625 (2019).

[25] J. P. Goss , R. Jones, S. J. Breuer, P. R. Briddon, and S. Öberg, The twelve-line $1.682 \mathrm{eV}$ luminescence center in diamond and the vacancy-silicon complex, Phys. Rev. Lett. 77, 3041 (1996).

[26] J. P. Goss, P. R. Briddon, M. J. Rayson, S. J. Sque, and R. Jones, Vacancy-impurity complexes and limitations for implantation doping of diamond, Phys. Rev. B 72, 035214 (2005).

[27] J. P. Goss, P. R. Briddon, and M. J. Shaw, Density functional simulations of silicon-containing point defects in diamond, Phys. Rev. B. 76, 075204 (2007).

[28] A. Gali and J. R. Maze, Ab initio study of the split siliconvacancy defect in diamond: Electronic structure and related properties, Phys. Rev. B. 88, 235205 (2013).

[29] G. Thiering and A. Gali, Ab initio magneto-optical spectrum of group-IV vacancy color centers in diamond, Phys. Rev. X 8, 021063 (2018).

[30] L. J. Rogers, K. D. Jahnke, M. W. Doherty, A. Dietrich, L. P. McGuinness, C. Müller, T. Teraji, H. Sumiya, J. Isoya, N. B. Manson, and F. Jelezko, Electronic structure of the negatively charged silicon-vacancy center in diamond, Phys. Rev. B 89, 235101 (2014).

[31] C. Hepp, T. Müller, V. Waselowski, J. N. Becker, B. Pingault, H. Sternschulte, D. Steinmüller-Nethl, A. Gali, J. R. Maze, M. Atatüre, and C. Becher, Electronic structure of the silicon vacancy color center in diamond, Phys. Rev. Lett. 112, 036405 (2014).
[32] T. Lühmann, N. Raatz, R. John, M. Lesik, J. Rödiger, M. Portail, D. Wildanger, F.Kleißler, K. Nordlund, A. Zaitsev, J.F. Roch, A. Tallaire, J. Meijer, and S. Pezzagna, Screening and engineering of colour centres in diamond, J. Phys. D 51, 483002 (2018).

[33] H. Hofsäss and G. Lindner, Emission channeling and blocking, Phys. Rep. 201, 121 (1991).

[34] U. Wahl, Advances in electron emission channeling measurements in semiconductors, Hyperfine Interact. 129, 349 (2000).

[35] L. M. C. Pereira, A. Vantomme, and U. Wahl, Characterizing defects with ion beam analysis and channeling techniques, chapter 11 in Characterisation and Control of Defects in Semiconductors, edited by F. Tuomisto (The Institution of Engineering and Technology, Stevenage, UK, 2019), pp. 501-563.

[36] U. Wahl, J. G. Correia, A. Czermak, S. Jahn, P. Jalocha, J. Marques, A. Rudge, F. Schopper, J. C. Soares, and A. Vantomme, Position-sensitive Si pad detectors for electron emission channeling experiments, Nucl. Instrum. Methods Phys. Res., Sect. A 524, 245 (2004).

[37] S. Decoster, S. Cottenier, U. Wahl, J. G. Correia, and A. Vantomme, Lattice location study of ion implanted Sn and Snrelated defects in Ge, Phys. Rev. B 81, 155204 (2010).

[38] S. Decoster, S. Cottenier, B. De Vries, H. Emmerich, U. Wahl, J. G. Correia, and A. Vantomme, Transition metal impurities on the bond-centered site in germanium, Phys. Rev. Lett. 102, 065502 (2009).

[39] R. Catherall, W. Andreazza, M. Breitenfeldt, A. Dorsival, G. J. Focker, T. P. Gharsa, T. J. Giles, J. L. Grenard, F. Locci, P. Martins, S. Marzari, J. Schipper, A. Shornikov, and T. Stora, The ISOLDE facility, J. Phys. G 44, 094002 (2017).

[40] K. Johnston, J. Schell, J. G. Correia, M Deicher, H. P. Gunnlaugsson, A. S. Fenta, E. David-Bosne, A. R. G. Costa, and D. C. Lupascu, The solid state physics programme at ISOLDE: recent developments and perspectives, J. Phys. G 44, 104001 (2017).

[41] V. N. Fedoseyev, G. Huber, U. Köster, J. Lettry, V. I. Mishin, H. Ravn, V. Sebastian, and the ISOLDE Collaboration, The ISOLDE laser ion source for exotic nuclei, Hyperfine Interact. 127, 409 (2000).

[42] J. F. Ziegler, J. P. Biersack, and M. D. Ziegler. SRIM, the stopping and range of ions in matter, Lulu Press, Morrisville, North Carolina, 2009.

[43] See Supplemental Material at http://link.aps.org/supplemental/10.1103/PhysRevLett.125.045 301, which includes Refs. [44-46], for more details on EC experiments, implementation of many-beam calculations for diamond, data analysis procedure, background correction, and PL measurements.

[44] S. L. Dudarev, L. M. Peng, and M. J. Whelan, On the DoyleTurner representation of the optical potential for RHEED calculations, Surf. Sci. 330, 86 (1995).

[45] S. Agostinelli et al. (GEANT4 collaboration), GEANT4-A simulation toolkit, Nucl. Instrum. Methods Phys. Res., Sect. A 506, 250 (2003).

[46] E. Bourgeois, E. Londero, K. Buczak, J. Hruby, M. Gulka, Y. Balasubramaniam, G. Wachter, J. Stursa, K. Dobes, F. Aumayr, M. Trupke, A. Gali, and M. Nesladek, Enhanced photoelectric detection of $\mathrm{NV}$ magnetic resonances in diamond 
under dual-beam excitation, Phys. Rev. B. 95, 041402(R) (2017).

[47] J. U. Andersen, E. Bonderup, E. Laegsgaard, and H. Sorensen, Incoherent scattering of electrons and linewidth of planarchanneling radiation, Phys. Scr. 28, 308 (1983).

[48] E. David-Bosne, U. Wahl, J. G. Correia, T. A. L. Lima, A. Vantomme, and L. M. C. Pereira, A generalized fitting tool for analysis of two-dimensional channeling patterns, Nucl. Instrum. Methods Phys. Res., Sect. B 462, 102 (2020).

[49] Diamond (C), Debye temperature, heat capacity, density, hardness, melting point and related data, in Group IV Elements, IV-IV and III-V Compounds. Part b - Electronic, Transport, Optical and Other Properties, edited by O. Madelung, U. Rössler, and M. Schulz (Springer-Verlag, Berlin, Heidelberg, 2002).

[50] R. John, J. Lehnert, M. Mensing, D. Spemann, S. Pezzagna, and J. Meijer, Bright optical centre in diamond with narrow, highly polarised and nearly phonon-free fluorescence at room temperature, New J. Phys. 19, 053008 (2017).

[51] A. Stacey, T. J. Karle, L. P. McGuinness, B. C. Gibson, K. Ganesan, S. Tomljenovic-Hanic, A. D. Greentree, A. Hoffman, R. G. Beausoleil, and S. Prawer, Depletion of nitrogen-vacancy color centers in diamond via hydrogen passivation, Appl. Phys. Lett. 100, 071902 (2012).

[52] R. K. Defo, E. Kaxiras, and S. L. Richardson, How carbon vacancies can affect the properties of group IV color centers in diamond: A study of thermodynamics and kinetics, J. Appl. Phys. 126, 195103 (2019). 